\title{
L'État moral: religion, nation et Empire dans la Grande-Bretagne victorienne et I'Inde britannique
}

\author{
Peter van der Veer, Sylvie Bach
}

\begin{abstract}
The Moral State: Religion, Nation and Empire in Victorian Great Britain and British India The comparative study of the role played by religion in the emergence of nationalism in Great Britain and India underscores the political importance of evangelist movements that legitimised the "civilising mission" of the English by developing the notion of a "moral' State", a discourse that culminated in the final decades of the century in the idea of "racial superiority". A vector of English. Scottish and Irish nationalism,- religion was nevertheless a decisive impediment to integrating the various elements of society into the British nation-state. The comparison with India also shows the strengthening of the links between religion (Hinduism) and nationalism. But in this case, for reasons. stemming from the structure of colonial society itself, the connection culminated : in the institutionalisation of religious partition between Hindus and Muslims.
\end{abstract}

\section{Citer ce document / Cite this document :}

van der Veer Peter, Bach Sylvie. L'État moral: religion, nation et Empire dans la Grande-Bretagne victorienne et l'Inde britannique. In: Genèses, 26, 1997. Représentations nationales et pouvoirs d'Etat. pp. 77-102;

doi : https://doi.org/10.3406/genes.1997.1433

https://www.persee.fr/doc/genes_1155-3219_1997_num_26_1_1433

Fichier pdf généré le 14/05/2018 
D O S S I E R

Genèses 26, avril 1997, pp. 77-102

L'ÉTAT MORAL:

RELIGION, NATION

ET EMPIRE

DANS LA GRANDE-

BRETAGNE

VICTORIENNE ET

L'INDE BRITANNIQUE

\section{Introduction}

En 1988, quand les Musulmans britanniques réclamèrent de leur gouvernement de bannir The Satanic Verses de Salman Rushdie, ils découvrirent que la législation existante sur le blasphème n'interdisait pas les insultes au Prophète Mohammed. Elle s'appliquait uniquement au Christianisme. C'est en invoquant cet argument que le gouvernement rejeta leur demande. Le ministre des Relations raciales en métropole, John Patten, rédigea, par la suite, un document qui expliquait aux Musulmans et au grand public ce qu'il fallait entendre par l'expression «être britannique». Talal Asad a brillamment analysé les implications politiques des vues libérales exprimées dans ce texte ${ }^{1}$, dont l'un des objectifs principaux était de définir une «culture nationale commune». Selon Patten, ce fond commun se trouvait dans «notre démocratie et nos lois, la langue anglaise, et l'histoire qui a déterminé la Grande-Bretagne moderne $»^{2}$. Dans cet article, je souhaite m'attacher à deux éléments qui ont été occultés dans le discours de Patten sur le fait d' «être britannique »: le Christianisme et l'Empire. Il est, bien sûr, tout à fait compréhensible qu'un homme politique n'ait pas mentionné la religion chrétienne comme l'une des composantes essentielles de la culture britannique, au beau
1. Talal Asad, Genealogies of Religion, Baltimore, Johns Hopkins University Press, 1993.

2. Cité dans Talal Asad, ibid., p. 244. 


\section{O S S I E R}

Représentations nationales et pouvoirs d'État

Peter van der Veer L'Etat moral:

religion, nation et empire

dans la Grande-Bretagne victorienne et l'Inde britannique milieu de «l'affaire Rushdie». Il n'en est pas moins vrai que les lois auxquelles Patten faisait allusion contiennent une disposition sur le blasphème qui ne protège que la sensibilité chrétienne. Qui plus est, personne ne peut douter du fait que la religion chrétienne ait été un élément crucial dans l'histoire qui a façonné la Grande-Bretagne. De la même façon, ce document présuppose qu' "être britannique » n'a rien à voir avec l'Empire. Autrement dit le conflit de valeurs, tel qu'il s'est manifesté dans l'affaire Rushdie, est vu comme un problème nouveau, apporté en Grande-Bretagne par l'immigration; qui n'a de rapport avec l'Empire que dans la mesure où les immigrants viennent de l'ancien monde colonial, illustrant en quelque sorte une nouvelle version de "l'Empire contre-attaque». Néanmoins, il serait tout à fait possible de soutenir que les arguments de Patten (prônant l'acceptation d'une culture nationale commune), aussi bien que ceux des chefs musulmans (qui appelaient à une neutralité religieuse de l'État, concrétisée par la protection accordée aux croyances de tous les groupes religieux), ont leurs racines dans la même histoire impériale, illustrant simplement les deux faces opposées du processus de colonisation. On dit parfois que les Britanniques ne sont pas conscients de leur histoire, parce qu'elle a eu lieu ailleurs. À mon avis, le lien avec l'Empire est effectivement un thème que les historiens de la Grande-Bretagne et, à plus forte raison, les hommes politiques britanniques ont trop rarement pris en considération, et sur lequel ils ont trop rarement réfléchi. Les historiens de l'Inde sont bien plus conscients du lien impérial, mais ont tendance à ignorer le cours des événements dans la métropole, par peur de faire de l'histoire de la colonie un simple post-scriptum de l'histoire de l'Europe. Je tâcherai ici de mettre au jour certaines similarités et différences structurelles entre le développement de la religion et du nationalisme en Grande-Bretagne et en Inde. Je dois cependant avertir le lecteur que cet article n'est pas écrit (comme c'est généralement le cas) in medias res, mais au tout début d'une nouvelle recherche. Je souhaite insister sur le fait que les positions que j'y défends constituent tout au plus une esquisse et un stade préliminaire dans un projet à plus long terme.

Selon moi, la relation cruciale à analyser ici est celle entre État, nation et religion, L'État moderne est un Étatnation, et le trait d'union indique qu'il faut à l'État moderne une nation, et vice versa. Bien que la Grande- 
Bretagne et l'Inde soient maintenant toutes deux des États-nations, pendant la période coloniale, seule la Grande-Bretagne était dans ce cas, l'Inde n'étant encore qu'une colonie. Ce constat semble indiquer, au minimum, l'existence d'un décalage entre le moment où la GrandeBretagne s'est constituée en État-nation colonial et le moment où l'Inde colonisée est devenue à son tour un État national, lui-même issu, peut être, de la colonisation. Il ne faut pas oublier, néanmoins, que la nation est une formation historique qui date du $\mathrm{XIX}^{\mathrm{e}}$ siècle, si bien que ce décalage historique est relativement mineur. Pour m'exprimer autrement, je dirai que c'est au moment où la Grande-Bretagne colonise l'Inde, que l'Angleterre colonise la «Grande-Bretagne", essayant d'unifier ce qui n'est pas encore (et ne sera que partiellement) le RoyaumeUni. Aujourd'hui encore, en Irlande du Nord et en Écosse, nous pouvons voir les conséquences historiques de ce processus. Sans vouloir m'attarder sur ce point, je voudrais seulement souligner le fait que cette notion de décalage temporel, fondée sur l'idée que le colonialisme aurait exporté des esquisses de l'État-nation vers des sociétés moins développées, risque de nous faire oublier que l'État-nation est issu d'un long processus de différenciation. On peut être ainsi conduit à ne pas voir que ce processus a impliqué la Grande-Bretagne et l'Inde simultanément, au cours de la même période historique. Que la religion ait été un facteur important dans l'émergence du sujet moderne ne semblera pas trop étrange à quiconque est familier avec la théorie weberienne de l'éthique protestante. En revanche, il peut sembler plus surprenant que la religion ait joué un grand rôle dans l'émergence de l'espace public moderne, surtout si l'on tient compte du fait qu'au XIX $X^{\mathrm{e}}$ siècle, ce n'est pas seulement le Protestantisme qui est "nationalisé», mais aussi le Catholicisme et beaucoup d'autres religions, telles que l'Islam et l'Hindouisme en Inde. On peut immédiatement objecter à cette affirmation que le Protestantisme est devenu la religion nationale de l'Angleterre et des Pays-Bas dès le $\mathrm{XVI}^{\mathrm{e}}$ siècle. À l'encontre de cette évidence, je voudrais plutôt suggérer qu'au début de l'époque moderne, il existe, dans ces pays, des Églises d'État protestantes mais qu'il n'y pas de religion nationale, du fait que les États-nations ne sont pas encore constitués. Autrement dit, c'est aux XVIII ${ }^{\mathrm{e}}$ et $\mathrm{XIX}^{\mathrm{e}}$ siècles que commencent à se produire de profonds changements au sein de la religion, qui affectent son organisation, son impact, sa portée. Ces changements doivent 
D O S S E R

Représentations nationales et pouvoirs d'Etat

Peter van der Veer L'Etat moral:

religion, nation et empire

dans la Grande-Bretagne

victorienne et l'Inde britannique
3. Pour les termes religieux, nous avons traduit Evangelicalism par évangélisme, Evangelicalist par évangéliste, Evangelical par évangélique, Roman Catholicism par Catholicisme romain et Roman Catholic par catholique romain (NDT)

4. John Wolffe, God and Greater Britain. Religion and National Life in Britain and Ireland 1843-1945, London, Routledge, 1995, p. 22. être mis en relation avec le développement de ce phénomène à trait d'union, qu'est l'État-nation. L'idée implicite sur laquelle s'appuie l'argumentation qui précède est que «le sujet moderne» s'est constitué en même temps que «l'espace public moderne». En conséquence, la religion a joué un rôle important non seulement dans la formation de la «conscience individuelle» et du «comportement civilisé », mais aussi dans la création de la sphère publique. Dans le reste de cet article, j'examinerai la «nationalisation» de la religion en Grande-Bretagne et en Inde. J'espère montrer que les événements qui se sont produits dans la métropole et ceux qui ont eu lieu dans la colonie ont d'importants points communs, mais qu'il y a aussi entre eux des différences substantielles qui tiennent à la manière dont État, nation et religion ont été articulés dans ces deux parties de l'Empire.

\section{L'État moral en Grande-Bretagne}

Dans la Grande-Bretagne du XIX ${ }^{\mathrm{e}}$ siècle, deux événements religieux majeurs vont jouer un rôle décisif dans les liens qui s'établissent alors entre religion et nationalisme. Le premier est l'énorme poussée de l'Évangélisme ${ }^{3}$ et son impact sur la culture religieuse de la Grande-Bretagne dans son ensemble. Le second est l'inclusion des Catholiques dans la nation et leur affranchissement. Prenons d'abord l'Évangélisme. On considère généralement que c'est dans la première moitié du XVIII ${ }^{\mathrm{e}}$ siècle, avec John Wesley, que débute le Renouveau Évangélique (Evangelical Revival). Mais celui-ci a connu une seconde période importante dans la dernière décennie du XVIII ${ }^{\mathrm{e}}$ siècle qui se prolonge jusqu'au XIX ${ }^{\mathrm{e}}$ siècle ${ }^{4}$. Le développement des mouvements évangéliques dans la première moitié du $\mathrm{XIX}^{\mathrm{e}}$ siècle est spectaculaire, mais plus important encore que leur nombre est l'impact considérable qu'ont eu ces mouvements sur des groupes religieux et des individus de toutes sortes. L'expansion évangélique coïncide largement avec la révolution industrielle. C'est ce qui explique la diversité des explications privilégiant les causes plus ou moins économiques, qui en ont été données, depuis Élie Halevy jusqu'à Edward Thompson. Toutes ces analyses ont fait l'objet, par la suite, de sérieuses critiques, mais cela n'est pas ici mon propos. Quelles qu'aient pu être les causes de ces mouvements, il est important de noter, pour le problème qui nous occupe ici, que l'Évangélisme appe- 
lait à la fois à la conversion intérieure des individus et à l'action extérieure dans le but de convertir les autres. Des prédicateurs itinérants et plus tard des sociétés bibliques et des sociétés missionnaires eurent un impact profond sur un large public. Nous avons ici affaire à un puissant mouvement civilisateur et éducatif, qui s'efforce de transformer la vie personnelle des gens. L'importance de l'Évangélisme dans la construction de l'individu moderne, civil et travailleur, ne fait aucun doute. En même temps, ces mouvements ont eu un impact considérable sur la vie politique. Le terme d' «Évangélisme» recouvre, il est vrai, toute une gamme d'idées et d'attitudes différentes, mais la campagne pour l'abolition de l'esclavage dans les premières décennies du XIX ${ }^{\mathrm{e}}$ siècle montre qu'au-delà de cette diversité, l'Évangélisme a pu être porteur d'un puissant message politique. C'est à ce niveau que l'on peut saisir comment il s'est tourné vers l'Empire. Les propos de William Wilberforce, l'un des chefs de la secte évangélique Clapham, sont à cet égard éloquents:

«Je considère qu'il est de mon devoir de commencer à délivrer ces pauvres créatures des ténèbres et de la dégradation dans lesquelles elles se trouvent, non seulement par égard pour leur bien-être [...], mais aussi parce que je suis absolument persuadé que si les colons, mais aussi nous-mêmes, nous n'accomplissons pas cette tâche, nous en subirons les conséquences. La justice et la punition divines se manifestent sous la forme de conséquences naturelles et c'est pourquoi je m'attends à ce que des malheurs se produisent ${ }^{5}$.

David Brion Davis suggère que l'abolition de la traite des esclaves en 1807 et de l'esclavage en 1833 furent de «véritables rituels», évoquant des fantasmes de mort et de renaissance, "ayant pour fonction de revitaliser le Christianisme et d'expier une faute nationale ${ }^{6}$. Ces attitudes envers le reste du monde étaient nouvelles et profondément modernes. Jusqu'à la dernière décennie du $\mathrm{XVIII}^{\mathrm{e}}$ siècle, c'est à peine si les missions à l'étranger suscitaient de l'intérêt. Mais les années 1790 à 1800 constituent des années-charnières, comme l'illustre au plus haut point le titre du livre de William Carey, An Enquiry into the Obligation of Christians, to Use Means for the Conversion of the Heathens ${ }^{7}$. Un grand nombre de sociétés missionnaires sont fondées à ce moment-là, y compris les célèbres LMS et CMS. Toutes ces sociétés se considèrent comme engagées dans une bataille contre l'idolâtrie et chargées du salut des âmes païennes. Non seulement on estime que ces âmes iront en enfer, si elles ne sont pas sauvées, mais
5. Cité dans Boyd Hilton, The Age of Atonement. The Influence of Evangelicalism on Social and Economic Thought, 1795-1865, Oxford, Clarendon Press, 1988, pp. 209-210.

6. Cité par Boyd Hilton, op. cit., p. 210.

7. «Une enquête sur l'obligation des Chrétiens d'utiliser des moyens pour la conversion des païens », 1792. 


\section{O S S E R}

Représentations nationales et pouvoirs d'État

Peter van der Veer L'Etat moral:

religion, nation et empire

dans la Grande-Bretagne

victorienne et l'Inde britannique
8. Cf. Brian Stanley, "Christian responses to the Indian mutiny of 1857", in Studies in Church History 20: The Church and War, W.J. Shiels (ed.), Oxford, Basil Blackwell, 1983, pp. 277-291, (en particulier p. 278).

9. Cf. Lynn Zastoupil, John Stuart Mill and India, Stanford, Stanford University Press, 1994.

10. "De la constitution de l'Église et de l'Etat ", 1829.

11. "L'Etat dans ses rapports avec l'Eglise », 1838. on en vient à considérer qu'il est du devoir d'un Chrétien de les sauver. On ne pourra jamais savoir jusqu'à quel point l'imagination chrétienne en Grande-Bretagne fut nourrie de cette imagerie de pauvres Hindous, de Musulmans, et d'autres êtres perdus pour l'éternité. Ce que nous savons, c'est qu'aux rassemblements des sociétés missionnaires organisés dans les provinces pour commémorer leurs anniversaires entre 1838 et 1873, un orateur sur deux était originaire d'Inde ${ }^{8}$. Il ne peut y avoir de doute sur le fait que les activités évangéliques simultanées des sociétés bibliques, des sociétés missionnaires, et des écoles du dimanche ont favorisé l'émergence d'une prise de conscience dans le public concernant l'existence d'un monde à part et d'un devoir impérial incombant aux Chrétiens britanniques dans l'Empire.

Dans le courant dominant de l'Évangélisme, l'enthousiasme religieux fut canalisé vers l'activité publique, disséminant les valeurs de la classe moyenne au sein d'une population plus large. En gros, il ne semble pas pertinent de voir dans l'Évangélisme un mouvement anti-rationaliste. Il s'agit plutôt d'un mouvement qui essaye de combiner la pensée rationnelle et le «sentiment » religieux, l'entendement et la sensibilité. C'est une raison parmi d'autres qui m'incite à l'interpréter plutôt comme un mouvement nationaliste typique, essayant de combiner la philosophie des Lumières et le Romantisme. Certes, il existe toujours un débat entre les libéraux utilitaristes (utilitarian liberals) et l'Évangélisme. Mais ces deux courants se rejoignent sur un terrain commun, comme le prouve la manière dont John Stuart Mill s'est efforcé de prendre ses distances à l'égard de l'hyper-rationalisme de son père ${ }^{9}$. L'Évangélisme visait à convertir les gens en vue d'une existence moralement inspirée, où la conscience individuelle des péchés et leur expiation deviennent les mots d'ordre d'une nation qui se donne une mission. Gladstone constitue un exemple intéressant de l'association du libéralisme et du moralisme évangélique. Élevé dans une famille évangélique dévote, au début de sa carrière il subit l'influence du livre du poète et philosophe Coleridge, On the constitution of Church and State ${ }^{10}$. Pour défendre l'Église en place dans la période qui suivit l'émancipation catholique, il écrit un livre, intitulé The State in its relations with the Church"l, dans lequel il accorde à l'État une conscience qui transcende celle des individus. Dans ce traité, non seulement il se fait l'avocat d'un lien étroit 
entre l'Église et l'État, mais il attribue également à ce dernier de grandes qualités morales. Comme Gladstone est devenu ultérieurement un partisan des droits des dissidents et des Catholiques, on a affirmé qu'il avait totalement renié ses conceptions antérieures ${ }^{12}$. Je crois plutôt qu'il s'agit chez lui d'une évolution qui l'a conduit à abandonner la conception pré-moderne de l'Église publique au profit d'une définition de l'État-nation dans laquelle ce n'est pas la bureaucratie étatique, mais la conscience individuelle et nationale qui priment. Ce qui demeure constant ici, c'est l'idée de la nature morale et religieuse de l'activité politique. Au lieu d'exclure les autres de cette vie morale de la nation, il souhaite à présent les y inclure tous. Ce qui exige de renoncer à la notion strictement calviniste des «quelques élus» pour la remplacer par un universalisme moral qui étend la grâce à tous les habitants du monde. Nous nous trouvons ici devant la vision d'une Église nationale, ou d'une nation perçue comme une Église, dépassant les dimensions visibles, institutionnelles, de l'Église d'Angleterre.

Une telle fusion de l'Église et de l'État-nation était également une nécessité cruciale pour la mission civilisatrice que Thomas Arnold envisage dans The Principles of Church Reform ${ }^{13}$. Mais alors que celui-ci se demande encore s'il est souhaitable d'inclure les Catholiques romains (les barbares irlandais) et si les groupes dissidents ont une chance de rejoindre cette mission christianisatrice et civilisatrice, ces doutes sont rapidement balayés par de nouvelles réalités sur le terrain. La doctrine libérale de l'amélioration de la société correspond parfaitement à ce moralisme chrétien. De ce point de vue, il est intéressant de voir que Coleridge n'a pas seulement influencé Gladstone et Arnold, mais aussi le porte-parole principal des idées libérales au XIX ${ }^{\mathrm{e}}$ siècle, John Stuart Mill ${ }^{14}$. Les images évangéliques habituelles de la damnation et de la fin des temps ont fait place, chez Gladstone, à une vision libérale du progrès. Mais s'y ajoute la conviction que le progrès signifie l'amélioration chrétienne de la société et que c'est dans un tel progrès que l'on peut voir la main de Dieu. Ce mélange d'idées libérales et évangéliques conduit généralement à souligner le caractère moral du peuple anglais et à rappeler que la tâche qui lui incombe est de conduire le monde ${ }^{15}$. Cette conception du progrès et de la grâce pour tous ne se limite donc pas aux Îles britanniques.
12. Boyd Hilton,

The Age of Atonement, op. cit., p. 341.

13. "Les Principes de la Réforme de l'Église», 1833.

14. Lynn Zastoupil, op. cit., 1994.

15. Cf. Keith Robbins,

«Religion and identity in modern British history ", in Stuart Mews (ed);

Studies in Church History 18:

Religion and National Identity. Oxford, Basil Blackwell, 1982, pp. 465-489. 


\section{O S S I E R}

Représentations nationales et pouvoirs d'Etat

Peter van der Veer L'Etat moral:

religion, nation et empire

dans la Grande-Bretagne

victorienne et l'Inde britannique
Elle intègre aussi l'idée que «le fardeau de l'homme blanc» est d'apporter l'Évangile aux peuples coloniaux.

Le passage d'une vision anglicane exclusive de la nation à un nationalisme inclusif, est reflété dans l'autre événement majeur de cette période, l'émancipation des Catholiques. L'Angleterre du XVIII ${ }^{\mathrm{e}}$ siècle avait surtout été un État protestant, mais la création de l'État-nation britannique exigeait l'inclusion de la minorité catholique. Il existait déjà un imposant passé d'hostilité anti-catholique en Angleterre, qui avait eu pour résultat l'exclusion des Catholiques de la plupart des domaines de la vie publique. À partir de 1800 , le Catholicisme romain, comme l'Évangélisme, connaissent un remarquable essor dû à la fois, dans le cas de l'Angleterre, à l'augmentation du nombre des Catholiques anglais et de l'énorme afflux des immigrants irlandais. En Irlande, le développement de l'activité catholique romaine, se traduit, en 1795, par la fondation, à Maynooth, d'un collège ayant pour but de former des prêtres. Mais le Catholicisme, comme l'Évangélisme, exercent aussi une influence en dehors de leur propre communauté religieuse. Cela est particulièrement visible dans le Mouvement d'Oxford (aussi appelé mouvement des "Tractarians») qui, à partir de 1833, contribue à accentuer le caractère catholique de l'Église d'Angleterre, donnant naissance à ce qui est alors appelé l'Anglo-catholicisme. En 1845, John Henry Newman (1801-1890), l'une des sommités de ce mouvement, remplace le terme «anglo" par celui de «romain». Il deviendra cardinal de l'Église catholique romaine en 1879. Les Évangéliques perçoivent l'augmentation du nombre des Catholiques romains comme une menace, sentiment exacerbé par la peur compréhensible que leur inspire «l'ennemi de l'intérieur» que constitue pour eux le mouvement d'Oxford. De 1820 à 1830, la lutte politique se focalise sur le droit des Catholiques romains à siéger au Parlement unifié de Grande-Bretagne et d'Irlande, droit qui leur a été concédé en 1829 par l'Émancipation. Non seulement les Catholiques romains sont désormais autorisés à faire partie de la nation, mais cette ouverture concerne également les dissidents, dont les incapacités civiles sont révoquées par les Test and Corporation Acts en 1828. Il faut lire les ouvrages de Coleridge, d'Arnold et de Gladstone à la lumière de ces événements, car ceux-ci ont contribué, sans aucun doute, à transformer le caractère religieux et politique de la société britannique de 
manière décisive. L'affranchissement de la minorité catholique dans les îles britanniques n'a pas été, néanmoins, suffisant pour empêcher le développement d'un lien très fort entre Catholicisme romain et nationalisme irlandais ${ }^{16}$. Ce lien apparaît très clairement lors de la Repeal agitation en 1843, au cours de laquelle le clergé catholique-romain et les nationalistes irlandais travaillent de concert pour attaquer l'union législative entre la Grande-Bretagne et l'Irlande. Ce mouvement, soutenu par tout un réseau d'organisations catholiques romaines, rencontre un immense succès populaire. Il n'est pas exagéré de voir dans le nationalisme irlandais l'exemple le plus parfait du nationalisme religieux en Grande-Bretagne. L'émancipation des Catholiques n'a donc pas réussi à attirer les Irlandais catholiques dans la nation britannique, car celle-ci conserve un caractère anglais trop accentué. De la même façon, les Presbytériens écossais ne sont pas immédiatement enclins à faire partie de la nation anglaise/britannique; comme le montre l'épisode de la Disruption, en 1843, qui voit la moitié des membres du clergé quitter l'Église établie pour former l'Église libre d'Écosse. Comme en Angleterre, l'Évangélisme travaille ici à promouvoir la cause du nationalisme, mais cette fois, il s'agit du nationalisme écossais. La motivation principale qui a conduit à la formation de l'Église écossaise, coïncide avec le besoin ressenti par les Évangéliques de se rapprocher des «gens». C'est la raison pour laquelle la Disruption s'accompagne de sentiments anti-anglais (encore très marqués de nos jours), qui se reflètent aussi bien dans l'opposition à Westminster que dans le rejet des propriétaires anglicisés. Beaucoup moins fort qu'en Irlande, le nationalisme en Écosse apparaît donc lui aussi, imprégné de religiosité. On pourrait peut être dire la même chose à propos du lien entre le nationalisme linguistique gallois et la religion «non-conformiste».

L'Émancipation catholique a détruit toute les illusions que des gens comme Thomas Arnold avaient pu entretenir sur la Grande-Bretagne comme nation protestante. Les sentiments anti-catholiques qui se font jour au sein de la majorité protestante n'ont pas empêché les Catholiques-romains de devenir la plus grande Église d'Angleterre au vingtième siècle ${ }^{17}$. En même temps, la constitution en nation de la «Plus Grande-Bretagne», incluant l'Irlande, s'est avérée impossible, à cause du succès qu'a rencontré l'alliance du Catholicisme romain et du
16. Cette section s'appuie en grande partie sur l'examen des nationalismes irlandais, écossais et gallois dans l'ouvrage de John Wolffe, God and Greater Britain, op. cit.

17. Ibid., p. 69. 


\section{O S S I E R}

Représentations nationales et pouvoirs d'Etat

Peter van der Veer L'Etat moral:

religion, nation et empire dans la Grande-Bretagne victorienne et l'Inde britannique
18. Ibid., p. 37.

19. 1864.

20. John Wolffe, The Protestant Crusade in Great Britain, 1829-1860, Oxford, Clarendon Press, 1991.

21. Ibid., p. 309.

22. Cité dans K. Robbins, «Religion and identity in modern British history", in Stuart Mews (ed.) Studies, op. cit., p. 471. nationalisme irlandais. Si l'anti-Catholicisme est très présent dans le mouvement évangélique, il faut insister sur le fait que le Catholicisme et l'Évangélisme ont connu tous les deux une importante expansion dans la première partie moitié du $\mathrm{XIX}^{\mathrm{e}}$ siècle, grâce à une dynamique alimentée par leurs rivalités. Ces deux mouvements se sont développés simultanément et ont essayé de dominer une sphère publique naissante dont l'existence même rendait possible le nationalisme. Il est utile, pour comprendre le Réveil évangélique et le Renouveau catholique romain, de les considérer comme deux mouvements liés l'un à l'autre, mais qui ont trouvé une bonne partie de leur dynamisme dans leur rivalité ${ }^{18}$. Sur ce point, il est intéressant de noter que l'Évangélisme, malgré son anti-Catholicisme, a suscité la plus célèbre conversion au Catholicisme du XIX ${ }^{\mathrm{e}}$ siècle, celle de John Henry Newman, comme il l'a franchement reconnu lui-même dans son Apologia Pro Vita Sua ${ }^{19}$. À partir des années 1830 jusque dans les années 1860, l'anti-Catholicisme et l'anti-ritualisme dans l'Église anglicane sont les thèmes majeurs de ce que John Wolffe a appelé la «Croisade protestante ${ }^{20}$. Celle-ci donne lieu à une agitation généralisée, mobilisant les Protestants aussi bien que les Catholiques. Une fois encore, je souhaiterais qu'on les examine ensemble. Les Évangélistes comme les Catholiques sont soucieux de mettre en avant leur nationalisme. Les Protestants en particulier se plaisent à souligner leur lien avec cet important symbole du nationalisme impérial qu'est la reine Victo$\mathrm{ria}^{21}$. Tandis que les Catholiques irlandais mettent évidemment en relief leur caractère irlandais, les Catholiques anglais s'efforcent de prendre encore plus nettement leurs distances à l'égard des déclarations d'allégeance au Pape, qui apparaissent comme anti-nationales.

Selon moi, ces deux mouvements ont fortement contribué à la naissance d'un nationalisme impérial et missionnaire, caractérisé par l'attribution de qualités nationales supérieures à la race dominante. C'est le thème de la nation qui se donne une mission. Ainsi que Michael Creighton, évêque anglican de Londres, l'affirme au début du siècle, «la question de l'avenir du monde est celle de l'existence de la civilisation anglo-saxonne sur une base religieuse ${ }^{22}$. Il est clair que c'est à l'Église d'Angleterre que Creighton pense lorsqu'il parle de «la conquête du monde", mais à mon avis, la diversité religieuse est impliquée dans la notion des devoirs d'une race 
supérieure. Dans la deuxième moitié du XIX $\mathrm{X}^{\mathrm{e}}$ siècle, la notion de supériorité raciale est fondée, dans une large mesure, sur l'idée de comparaison; la civilisation étant définie par son antithèse, la barbarie ou la sauvagerie. Les rivalités internes, les animosités et les conflits à l'intérieur du monde chrétien britannique s'effacent pour devenir la toile de fond de ce qui apparaît progressivement comme la principale différence; celle qui oppose la civilisation chrétienne britannique et la barbarie des peuples colonisés. L'affirmation biblique selon laquelle l'humanité est une et a pour origine un seul couple d'humains dans le Jardin d'Eden, tout comme les notions d'identité et d'égalité universelles défendues par les Lumières, laissent rapidement la place, dans la seconde moitié du XIX ${ }^{\mathrm{e}}$ siècle, à l'idée d'une différence raciale radicale ${ }^{23}$. Des philologues comme Renan et Max Müller assimilent race et langue; Renan affirmant le droit des races supérieures à coloniser les races inférieures. Là où Thomas Arnold avait été très attentif à la relation entre religion et nation, son fils Matthew Arnold, l'auteur de Culture and Anarchy, adapte cette préoccupation en privilégiant une définition racialisée de la culture. La vision arnoldienne de la culture conserve ainsi son inspiration religieuse. Il est important de noter qu'Arnold était Inspecteur des Écoles et qu'à ce titre il était responsable de l'éducation des Britanniques dans le cadre de cette nouvelle mission de la nation, définie en termes de race. La science moderne apporte son soutien à la construction idéologique de la culture nationale, centrée sur la langue et la race, tandis que la culture des colonisés devient un objet d'études académiques, disposant désormais de sa propre chaire universitaire ${ }^{24}$. Dans la deuxième partie du XIX ${ }^{\mathrm{e}}$ siècle, peu à peu, la race prend le pas sur la religion et devient l'élément dominant du nationalisme britannique.

\section{La mission coloniale en Inde}

Au début du XIX ${ }^{\mathrm{e}}$ siècle, au sein de l'East India Company, l'un des principaux débats sur la politique à suivre oppose les "Orientalistes», qui affirment que la Compagnie doit continuer sa politique de soutien aux institutions religieuses et éducatives indigènes, et les «Anglicistes" qui considèrent qu'il n'y a pas grand-chose de valable dans ces institutions indigènes, et qu'elles doivent être remplacées par les institutions plus civilisées et plus
23. Robert Young, Colonial Desire, Hybridity in Theory, Culture and Race, New York, Routledge, 1995, p. 47.

24. Cf. R. Young, op. cit., p. 71. 


\section{O S S I E R}

Représentations nationales et pouvoirs d'Etat

Peter van der Veer L'Etat moral:

religion, nation et empire

dans la Grande-Bretagne victorienne et l'Inde britannique

25. «Rapport sur l'Education indienne».

26. Cf. Gauri Viswanathan, Masks of Conquest. Literary Study and British Rule in India, New York, Columbia University Press, 1989, p. 102.

27. Geoffrey Oddie, Hindu and Christian in South-East India, London, Curzon Press, 1991, p. 57. avancées de l'Angleterre. Ce débat, certes complexe, a été gagné, d'une manière plus ou moins décisive, par les Anglicistes, lorsque Minute on Indian Education ${ }^{25} \mathrm{de}$ Thomas Babington Macaulay, publié en 1835, a été accepté comme le fondement officiel de la politique de la Compagnie. Dans cette bataille, les Anglicistes sont soutenus par les Évangélistes. Ces derniers - tout comme les membres de la secte Clapham (William Wilberforce, Zachary Macaulay, John Venn, Samuel Thornton, Charles Grant) qui ont joué un grand rôle dans la campagne anti-esclavagiste - sont indignés par le soutien que la Compagnie a apporté à l'Hindouisme et à l'Islam en Inde. Ils rejoignent les Anglicistes utilitaristes dans leur mépris pour les institutions indigènes et les littératures indiennes. William Wilberforce affirme, devant le Parlement anglais, que les Orientalistes sont aussi indifférents au Christianisme que les révolutionnaires français, dont les actions sont considérées avec horreur ${ }^{26}$. En conséquence, la Compagnie doit non seulement autoriser les missionnaires à travailler en Inde (ce qu'elle fait, après 1813), mais elle doit également cesser de soutenir les institutions indigènes.

Dans les premières décennies du XIX ${ }^{e}$ siècle, la Compagnie patronne toujours des temples et des festivals hindous, surtout dans le Sud. Sous la forte pression des Évangélistes, elle est contrainte de renoncer à cette politique. Ce qu'elle fait après beaucoup d'hésitations. En 1838, on voit encore apparaître en Angleterre, un comité qui se donne pour but de «diffuser des renseignements relatifs aux liens qu'entretient le gouvernement de l'East India Company avec les systèmes d'idolâtrie et de superstition des indigènes», et qui cherche à "promouvoir la dissolution de ce lien ${ }^{27}$. Il faut néanmoins considérer cette attitude comme une sorte de repli précédant une politique active qui conduit les Britanniques à mettre sur pied toutes sortes de systèmes et de comités pour contrôler les fondations religieuses. Ces comités deviennent des arènes importantes, à partir desquelles les Hindous et les Musulmans entreprennent d'organiser la sphère publique. Nous avons là un autre exemple de la nouvelle politique coloniale de représentation qui prend la place des anciens réseaux de patronage auxquels la Compagnie avait participé pour favoriser ce qui restait son objectif principal: le commerce. Les Utilitaristes et les Évangéliques sont d'accord sur un point: les institutions religieuses de l'Inde 
doivent être démantelées et remplacées par la civilisation chrétienne. Mais ils divergent sur la manière d'apporter la civilisation aux indigènes. La neutralité religieuse avait été considérée comme essentielle, d'abord pour des considérations commerciales, puis pour asseoir l'autorité britannique en Inde. La Compagnie continue ensuite à s'opposer à l'idée d'un soutien direct aux projets des missionnaires. L'Anglican Society for the Propagation of the Gospel in Foreign Parts (établie en 1701) avait toujours été une Église coloniale formant les membres du clergé pour les Britanniques vivant dans les colonies, jusqu'à sa transformation dans les années 1830 à 1840 sous l'influence de l'Évangélisme ${ }^{28}$. À l'extérieur de la Compagnie, ce n'est qu'au XIX siècle qu'une importante activité missionnaire est développée parmi les indigènes, dans des cercles qui collectent de l'argent au sein du public britannique. Néanmoins, en dépit de la neutralité de la Compagnie, on voit apparaître des tentatives visant à réformer la société indienne par l'éducation, entreprise entièrement soutenue par les Anglicistes utilitaristes. C'est dans ce domaine que les missionnaires allaient devenir particulièrement actifs.

Quelles qu'aient été les dissensions entre Évangéliques et Utilitaristes, et elles étaient de taille, aucun d'entre eux n'aurait nié que la société civile et les formes de connaissance sur lesquelles elle était fondée ne fassent en dernière analyse partie intégrante de la civilisation chrétienne. Gauri Viswanathan a défendu avec force la thèse selon laquelle l'enseignement de la littérature anglaise «laïque», tel que le Rapport de Macaulay le recommandait, peut être considéré comme un déplacement de la valeur culturelle attribuée à la croyance et au dogme, vers le langage, l'expérience et l'histoire ${ }^{29}$. C'est précisément ce déplacement que l'on peut détecter dans les différences intellectuelles qui séparent, tout en les unissant, Matthew Arnold et son père Thomas, ainsi que Thomas Babington Macaulay et son père Zachary. En dépit de leurs divergences, ces individus se trouvent dans le même univers spirituel. Leurs différences ne portent pas sur la mission morale de l'État, mais sur des questions de politique. Les changements qui se produisent à ce niveau concernent tout autant la Grande-Bretagne que les Britanniques vivant en Inde. Pour les Évangéliques et les Utilitaristes, le monde ne se limite plus à l'Angleterre ou à la Grande-Bretagne. Les campagnes anti-esclavagistes
28. Cf. Brian Stanley, The Bible and the Flag. Protestant Missions and British Imperialism in the nineteenth and twentieth centuries,

Leicester, Apollos, 1990, p. 61.

29. G. Viswanathan, op. cit., p. 117. 


\section{O S S I E R}

Représentations nationales et pouvoirs d'Etat

Peter van der Veer L'Etat moral: religion, nation et empire dans la Grande-Bretagne victorienne et l'Inde britannique
30. Cf. Peter van der Veer, Religious Nationalism. Hindus and Muslims in India, Berkeley, University of California Press, 1994. ont sensibilisé le public britannique au rôle que doit jouer leur pays au sein d'un monde plus large, et chacun s'accorde pour considérer que ce rôle doit être compris en termes de réforme et d'élévation morale.

Malgré les efforts déployés par les Britanniques pour dissimuler les racines chrétiennes de leur politique coloniale derrière le masque de la neutralité religieuse, les «indigènes » colonisés n'ont pas été dupes. On mentionne souvent les grandes différences qui existaient entre les opérations des sociétés missionnaires en Inde et celles de l'État, mais ces différences se situent à l'intérieur d'un même projet colonisateur. Il est bien vrai que les officiers de la Compagnie et plus tard l'État colonial méprisaient les missionnaires et qu'il y avait, en général, un écart social sensible entre eux. Néanmoins, leurs préoccupations se rejoignent dans les domaines cruciaux de l'éducation et de la réforme, tout comme elles se rejoignent en métropole. La véritable différence se trouve, bien sûr, non pas entre l'État colonial et les missionnaires, mais entre la Grande-Bretagne colonisatrice et les Indiens colonisés. Tandis qu'en Grande-Bretagne, l'État se met à occuper, petit à petit, les espaces sociaux ouverts par les organisations religieuses, en Inde, ces espaces sont occupés par des organisations religieuses rivales de «sujets» indigènes. Leurs idées et leurs actions ne pouvaient être incorporées à une nation britannique caractérisée par sa civilisation chrétienne. Finalement, elles ont fini par s'opposer à l'État colonial, devenant du même coup, un vecteur du nationalisme indien. En dépit de la politique officielle de neutralité, les Britanniques interviennent dans tous les aspects de la religion et de la société indienne. Étant donné la nature du projet colonial, il n'y a, en fait, pas de choix et la rhétorique du «retrait», de la «laïcité», et de la «neutralité» ne sert qu'à tenter d'occulter par le discours, cette logique interventionniste. Même si je ne compte examiner ici que l'engagement britannique dans l'Hindouisme et ses conséquences, je dois dire, néanmoins, que les événements qui ont eu lieu dans l'Islam et le Sikhisme indien n'étaient pas fondamentalement différents ${ }^{30}$. La politique suivie par les Britanniques a déclenché toute une chaîne de réactions réformistes dans l'Hindouisme. Comme dans le cas du «réveil» évangélique en Grande-Bretagne, les facteurs en jeu sont extrêmement complexes et il n'est pas possible de ne voir dans la Réforme qu'une réaction au projet colonial. Je 
voudrais néanmoins attirer l'attention sur le fait que des organisations réformistes sont parvenues à constituer un espace public en utilisant des moyens qui rappellent les activités évangéliques en Grande-Bretagne. Pour illustrer ce point, j'examinerai brièvement d'une part, la constitution de la «spiritualité hindoue» dans le Brahmo Samaj et la mission Ramakrishna et, d'autre part, la construction de la «race aryenne» dans l'Arya Samaj.

L'un des premiers exemples de mobilisation hindoue s'opposant à la domination coloniale dans l'espace public, apparaît à l'occasion de l'abolition du sati (l'immolation de la veuve) par les Britanniques en 1829. Le sati était peut-être le signe le plus évident de la dépravation hindoue et de la supériorité morale chrétienne aux yeux des Évangéliques. C'est pourquoi, ils concentrent leurs attaques contre les institutions indigènes afin d'obtenir l'abolition de cette pratique. Ils réussissent à convaincre le gouverneur-général William Bentinck qui, plus tard, transformera en décrets les propositions avancées par les Anglicistes à propos de l'éducation indienne. Une statue en l'honneur de Bentinck, érigée peu de temps après son départ d'Inde en 1835, montre une scène de sati qui se déroule sous son regard sévère et qui contient une inscription, située à l'arrière du socle, soulignant que la Grande-Bretagne s'est désormais engagée à «élever le caractère moral et intellectuel» des sujets indiens ${ }^{31}$. Il serait évidemment facile de détecter, derrière ce moralisme évangélique, le fantasme sexuel des «hommes blancs sauvant les femmes brunes des hommes bruns $\gg 2$. La position prise par les citoyens «éclairés» de Calcutta apparaît plus importante encore que les actions des Évangéliques et les réactions du gouvernement. Rammohan Roy (1772-1833), parfois appelé «le père de la Renaissance bengalaise ", a beaucoup écrit sur ce sujet entre 1818 et 1832. En janvier 1830, Rammohan, accompagné de trois cents résidents de Calcutta, présente une pétition à Bentinck en faveur de règlements interdisant le sati. Rammohan rejette cette pratique en se fondant sur une lecture personnelle des textes sacrés hindous. Il distingue les sources qui font autorité (telles que les Veda) et les autres sources. Il est intéressant de remarquer qu'il ne se réfère à aucune des interprétations autorisées que les gourous érudit ont donné de ces sources. Il fonde entièrement ses analyses sur son jugement personnel, rationnel. C'est certainement un grand pas qui est franchi vers la
31. Cf. Thomas Metcalf, Ideologies of the Raj, Cambridge, Cambridge University Press, 1994, p. 95.

32. Gayatri Spivak, "Can the Subaltern Speak ", in Cary Nelson and Lawrence Grossberg (eds), Marxism and the Interpretation of Culture, Urbana, University of Illinois Press, 1988, pp. 271-313. 


\section{O S S I E R}

Représentations nationales et pouvoirs d'État

Peter van der Veer L'Etat moral:

religion, nation et empire dans la Grande-Bretagne victorienne et l'Inde britannique

33. Wilhelm Halbfass, India and Europe, Albany, SUNY Press, 1988, pp. 205-206.

34. Lata Mani, «Contentious traditions: The debate on Sati in Colonial India », in Kumkum Sangari and Sudesh Vaid (eds) Recasting Women, New Brunswick,

Rutgers University Press, 1990.

35. Ranajit Guha, «A Construction of Humanism in Colonial India ", Wertheim Lecture, Amsterdam, CASA, 1993.

36. 1838-1894. laïcisation de l'Hindouisme. Cela nous montre aussi à quel point l'autorité des textes sacrés eux-mêmes est devenue importante, puisque même un non-initié peut y faire référence, sans la médiation d'un interprète sacré. L'un des objectifs majeurs de Rammohan est d'abolir le caractère héréditaire, fondé sur le système des castes, des compétences exigées de ceux qui veulent étudier les $\mathrm{Veda}^{33}$. En suivant Lata $\mathrm{Mani}^{34}$, je voudrais suggérer que l'importance que le pouvoir colonial accorde à l'autorité de ces textes pour interpréter la tradition, s'explique par le développement des études orientalistes consacrées à ces sources. Intérêt qui explique l'affaiblissement progressif, dans l'Hindouisme, de la tradition orale au profit de l'écrit. J'ajouterai, cependant, que la primauté accordée au texte avait aussi été soulignée par les Évangéliques qui raillaient la pratique du sati. La position de Rammohan s'apparente donc à la fois aux modes de pensée orientalistes et protestants. Le privilège qu'il accorde à son propre jugement rationnel, fondé sur la lecture et la discussion, rend possible l'émergence d'un espace public ainsi qu'une certaine forme de débat public, au sens de Jürgen Habermas.

S'il faut insister sur le strict parallélisme que présente le développement des «religions rationnelles» indienne et européenne, nous devons avoir à l'esprit la différence cruciale qui les sépare: tandis que les Chrétiens d'Europe essayent d'universaliser leur tradition religieuse, les Hindous d'Inde font la même chose avec leur propre tradition. Ainsi se reproduit une opposition Hindou-Chrétien qui recouvre aussi l'opposition colonisé-colonisateur. Le colonialisme a fourni le cadre discursif dans lequel la religion rationnelle hindoue a émergé. Comme Ranajit Guha $^{35}$ l'a démontré, cela apparaît aussi clairement dans l'œuvre du penseur humaniste Bankimchandra Chattopadhyay $^{36}$, très influencé par Auguste Comte, mais qui se situe en dehors du cercle du Brahmo Samaj. Bankimchandra (lui aussi un brahmane) construit sa vision de "l'humanité» (manusyatva), comme beaucoup de penseurs européens, autour de la notion de la perfectibilité de l'homme. Mais à la différence des Européens, il estime possible de donner des exemples d' "homme idéal» (Adarsa Purush) dont la perfection doit être un objet d'émulation. Ces exemples sont pris dans l'histoire religieuse hindoue et placent au plus haut le dieu Krishna. L'homme le plus parfait est donc un dieu hindou. La 
question des Lumières sur la nature de l'homme trouve ainsi, dans le cadre colonial, une réponse spécifique, définie dans les termes du nationalisme religieux. L'intellectualisme des conceptions védiques et unitariennes des Brahmos a contribué, dans une large mesure, à leur isolement au sein de la société hindoue du Bengale. Dans ce cadre plus large, une espèce particulière de dévotions bengales Vaishnavite acquiert peu à peu de l'importance à partir du XVI ${ }^{\mathrm{e}}$ siècle. Ces dévotions mettent l'accent sur le dieu Krishna et sur des gourous qui descendent des disciples du grand gourou du $\mathrm{vI}^{\mathrm{e}}$ siècle, Chaitanya. Dans la deuxième moitié du XIX ${ }^{e}$ siècle, cette tradition de dévotions commence à exercer une influence considérable sur la religion rationnelle des Brahmos. Dans les années 1860, Keshabchandra Sen ${ }^{37}$, l'un des chefs Brahmo parmi les plus influents, introduit le chant comme forme de dévotion dans les réunions des congrégations $B r a h m o^{38}$. Il ne parle plus l'anglais, mais le bengali. Il s'installe dans les faubourgs ruraux de Calcutta et introduit un style de vie ascétique parmi ses adeptes. L'étape suivante semble avoir été sa rencontre avec son contemporain, le gourou Ramakrishna ${ }^{39}$, un des prêtres de la déesse-mère Kali à Calcutta. Dans ses deux journaux (l'un en anglais, l'autre en bengali), il présente Ramakrishna à un public plus vaste, comme un véritable saint dans la plus authentique tradition hindoue. Il fait ainsi de cet Hindou ascétique illettré un gourou acceptable pour les classes moyennes hindoues. Dans un livre récent sur le nationalisme, Partha Chatterjee ${ }^{40}$ décrit la rencontre de ces deux personnalités comme constitutive du «terrain commun», occupé par les classes moyennes naissantes, entre la philosophie européenne rationnelle et le discours religieux hindou. Selon lui, ce «terrain commun» a permis aux nationalistes anticolonialistes de diviser le monde en deux domaines: le monde extérieur, matériel, qui est dominé par la science occidentale, et le monde «intérieur», spirituel, qui est dominé par les valeurs hindoues.

La spiritualité de la civilisation hindoue, cependant, n'est pas seulement symbolisée par la maison, mais aussi par une action réformiste et politique, comme beaucoup plus tard dans l'action non-violente (satyagraha) de Gandhi. Le thème de l'opposition entre la spiritualité hindoue et le matérialisme occidental devient certainement le thème principal du discours nationaliste hindou à partir de cette période. Une étape majeure dans la popularisation
37. 1838-84.

38. David Kopf, The Brahmo Samaj and the Shaping of the Modern Indian Mind. Princeton, Princeton University Press, 1979.

39. $1836-86$.

40. Partha Chatterjee, The Nation and Its Fragments, Princeton, Princeton University Press, 1993. 


\section{O S S I E R}

Représentations nationales et pouvoirs d'État

Peter van der Veer L'Etat moral:

religion, nation et empire

dans la Grande-Bretagne victorienne et l'Inde britannique
41. 1863-1902.

42. Tapan Raychaudhuri, Europe Reconsidered, Delhi, Oxford University Press, 1988. des idées réformistes hindoues est franchie au moment où elles s'allient au nationalisme naissant. La «spiritualité hindoue » devait, en effet, être défendue contre les attaques de la modernité coloniale. Le fondateur de la Mission Ramakrishna, Vivekananda, peut être considéré comme l'interprète le plus important de la doctrine de la «spiritualité hindoue ${ }^{41}$. Vivekananda est un étudiant très brillant qui a été nourri de la pensée occidentale contemporaine. Il rejoint le Brahmo Samaj peu de temps avant sa rencontre avec Ramakrishna. Cette rencontre allait avoir un impact décisif sur le jeune Narendranath Datta qui adopte le nom de Vivekananda au moment où il prononce ses vœux d'ascétisme. Ainsi que Tapan Raychaudhuri le souligne, Vivekananda est "avant tout un mystique en quête de l'ultime réalité à l'intérieur d'une tradition indienne spécifique ${ }^{42}$. Cette tradition, il ne l'a pas acquise dans des textes d'érudition, terrain où il était lui-même un maître, mais grâce à la présence charismatique d'un gourou, Ramakrishna, dont les transes avaient d'abord été considérées comme une forme de "démence», avant que l'on y voie la preuve qu'il était "possédé » par la déesse. Il me semble que c'est l'articulation de la «religion rationnelle» brahmo avec le discours religieux de Ramakrishna qui a produit l'espèce particulière de «spiritualité hindoue» que Vivekananda s'est mis à propager. La stratégie typique de Vivekananda consiste à systématiser un ensemble disparate de traditions, pour les rendre accessibles à un public occidentalisé et défendable contre la critique occidentale, puis à l'incorporer au sein d'une «spiritualité hindoue», portée par la nation hindoue. Cette spiritualité est, selon lui, supérieure au «matérialisme occidental», apporté en Inde par une «nation britannique» agressive et arrogante. Sa principale réussite a été de reconstruire le projet visant à fonder la «spiritualité hindoue " autour d'une interprétation systématique des Vedanta (les Upanishads et la tradition constituée par leur interprétation). Ce projet, commencé avec Rammohan Roy, et qui avait produit un Hindouisme rationnel, est à présent combiné avec d'autres disciplines pour atteindre, en partant des traditions ascétiques, la perfection dans le cadre de ce que Vivekananda appelle les «vedanta pratiques». Le côté pratique inclut aussi la participation à la réforme sociale. Cette sorte d' "Hindouisme spirituel» allait être reprise plus tard par Mahatma Gandhi et Sarvepalli Radhakrishna, mais elle est aussi devenue l'un des principaux éléments du nationalisme hindou actuel. Un 
bon exemple de la construction de la spiritualité hindoue se trouve dans les efforts faits par Vivekananda pour intégrer les notions disparates qui appartenaient à la pratique ascétique, au sein d'un «système ancien de yoga» qui est à présent l'article le plus exporté de l'Inde sur le «marché de la spiritualité». Yoga est un terme sanskrit que l'on peut traduire par «discipline». Le texte classique est le Yoga Sutras de Patanjali, probablement composé vers le $\mathrm{v}^{\mathrm{e}}$ siècle de notre ère. Vivekananda systématise cette tradition dans une doctrine du salut, où il combine la pensée rationnelle, les idées de Patanjali sur la méditation, l'action sociale et la dévotion religieuse. C'est là une nouvelle doctrine, bien que Vivekananda ait insisté sur le fait qu'il s'agissait d'une «sagesse ancienne». On peut y voir une étape essentielle dans le processus qui a conduit à une présentation systématique de la "spiritualité hindoue» comme étant bénéfique, à la fois pour le corps et pour l'esprit. Le fait que le projet de Vivekananda ait reçu une impulsion majeure au moment où il a été accueilli avec enthousiasme en Europe et aux États-Unis mérite aussi d'être noté. Sa visite au Parlement mondial des Religions à Chicago en 1893 a fait de lui une célébrité aux ÉtatsUnis et par conséquent aussi en Inde. Son nouveau statut de gourou international a conforté ses vues sur la contribution que l'Inde pouvait apporter à la civilisation du monde. Le nationalisme est un élément majeur du message de Vivekananda. Il définit surtout son projet en termes de revitalisation de la nation hindoue. En 1897, il fonde un ordre ascétique, la Mission Ramakrishna, pour que les ascétes soient mis à la disposition de la cause nationaliste. L'autodétermination nationale, la réforme sociale et le réveil spirituel, tout cela est lié à ses yeux. La Mission Ramakrishna s'établit dans toute l'Inde et au-delà. Si elle ne s'est pas transformée en mouvement de masse, la rhétorique spiritualiste de Vivekananda a exercé une immense influence sur la manière dont les gourous $\mathrm{du} \mathrm{XX}^{\mathrm{e}}$ siècle en sont venus à communiquer leur message. Vivekananda a transformé le discours hindou sur l'ascétisme, la dévotion et le culte en un idiome nationaliste de «service à la nation" pour les hommes comme pour les femmes.

La construction de la «spiritualité hindoue» par Vivekananda a donné à la notion sacrificielle d' «autosacrifice » un sens nouveau inspiré à la fois des traditions hindoues de dévotion (bahkti) et des notions évangéliques de moralité féminine. Nous avons ici un mélange 


\section{O S S I E R}

Représentations nationales et pouvoirs d'Etat

Peter van der Veer L'Etat moral:

religion, nation et empire

dans la Grande-Bretagne victorienne et l'Inde britannique
43. Cf. Peter van der Veer, "Gender and Nation in Hindu Nationalism ", in Hans Antlov et Stein Tonneson (eds) Asian Forms of the Nation, London, Curzon Press, 1996, pp. 188-213.

44. Cf. B. Stanley, "Christian responses to the Indian mutiny of 1857 », op. cit. complexe, dans lequel la «féminité» est le signifiant de la «spiritualité hindoue», tandis que les femmes réelles doivent se sacrifier, en accord avec la conception victorienne de la «domesticité» et la conception hindoue de la dévotion totale due au mari. Ainsi l'abolition du sati par le gouvernement colonial a-t-elle déclenché toute une série de réactions hindoues qui ont eu une portée considérable, puisqu'elles ont entraîné, à leur tour, la formation d'une conception moderne de la spiritualité à travers laquelle la nation hindoue s'est trouvée définie. La question du genre était un enjeu essentiel dans la prohibition du sati et un élément crucial dans la définition de la «spiritualité hindoue », étant donné l'accent mis sur «la dévotion féminine et l'auto-sacrifice ${ }^{43}$. Avec la formation de l'«aryanisme hindou», ce sont les questions de race et de caste qui passent au premier plan. La mutinerie de Cipayes de l'armée du Bengale et la révolte qui s'ensuivit en Inde du Nord en 1857 , ainsi que sa répression en 1858 , ont contribué, dans une très large mesure, à la notion de différence raciale et religieuse entre colonisateurs et colonisés. Pendant cette période de grande anxiété, due à la crainte d'une perte de contrôle sur l'Inde, des histoires racontant les atrocités inhumaines infligées à des femmes et à des enfants britanniques ont circulé rapidement dans toute la Grande-Bretagne, confirmant l'idée déjà répandue de la barbarie des Indiens qui avait été mise en circulation lors de la description du sati. La répression de la révolte démontrait une fois pour toutes que les Britanniques appartenaient à une race supérieure. Les Évangéliques estimaient, cependant, que les Britanniques n'avaient pas pris suffisamment au sérieux la mission civilisatrice incombant à la race supérieure. Pour eux ces événements constituaient un jugement divin pour punir la GrandeBretagne des péchés qu'elle avait commis, en tant que nation. Ce sont les négligences de la Compagnie concernant la promotion de l'Évangile qui étaient considérées comme la faute la plus grave. C'est pourquoi, en GrandeBretagne, le dimanche 7 octobre 1857, un grand nombre d'Églises, à la fois anglicanes et non-conformistes, participent à la «journée d'humiliation nationale», proclamée par la Reine Victoria ${ }^{44}$. Dans les sermons, ce jour-là, presque tous les prédicateurs sont d'accord sur la nécessité d'effacer l'humiliation, de réprimer la révolte par des moyens militaires, et d'infliger un châtiment à la population indienne. Les qualités chrétiennes montrées par certains des officiers britanniques durant la révolte sont lon- 
guement exaltées, et, de la même manière, Henry Havelock attribue sa victoire à Fatehpur, «à la bénédiction accordée par Dieu à une très juste cause, la cause de la justice, de l'humanité, de la vérité et du bon gouvernement de l'Inde ${ }^{45}$. À plus long terme, cependant, la révolte a convaincu la plupart des officiers coloniaux que la conversion au Christianisme était une lutte laborieuse et a conforté l'idée que la neutralité religieuse était essentielle à la domination coloniale. Il était devenu difficile, pour eux, de concevoir que les barbares indiens pourraient devenir un jour les égaux des Chrétiens britanniques. Lord Canning renvoie l'Évangélique Herbert Edwardes, commissaire de Peshawar, parce qu'il est, selon lui, «exactement ce que Mahomet aurait été s'il était né à Clapham au lieu de la Mecque ${ }^{46}$. La différence raciale entre les Britanniques et les colonisés et parmi les colonisés eux-mêmes devient à la fois l'explication et la légitimation de la domination coloniale.

Bien que ce racisme aux forces toutes neuves coïncide avec la montée du nationalisme racial dans la métropole, sur le plan de la pensée scientifique, l'idée que les castes supérieures de l'Inde appartiennent à la même race aryenne que les Britanniques est alors largement acceptée. En Inde, il fallait combiner l'idée de race avec celle de culture ou de civilisation pour expliquer pourquoi les Britanniques, en tant que "petits frères» de la famille aryenne, devaient guider leurs «grands frères» vers la civilisation. C'est toujours la différence religieuse qui constitue ici la grande référence culturelle. L'histoire de la race aryenne en Inde est présentée comme l'histoire d'un déclin, causé par divers facteurs, tels que le mélange des races ou le climat, mais surtout par la barbarie inhérente au polythéisme hindou. Les idées raciales ne sont pas seulement théoriques. Elles influencent aussi les modes de recrutement dans l'armée, qui accepte les races «martiales», comme les Sikhs Punjabi et exclut les races "efféminées», comme les Bengalis. Une autre distinction importante est faite entre les "Aryens» et les «Drâvidiens» en Inde du Sud. C'est en partant de cette différence que le missionnaire Robert Caldwell a construit ses théories linguistiques et ethnologiques sur les langues et les peuples drâvidiens. Dans la deuxième moitié du $\mathrm{XIX}^{e}$ siècle, il développe le mythe de l'invasion aryenne (brahmanique) de l'Inde du Sud. La sujétion du peuple drâvidien au système des castes hindou étant, selon lui, une
45. Ibid., p. 288.

46. Cité dans T. Metcalf, Ideologies of the Raj, op. cit., p. 48. 


\section{O S S I R}

Représentations nationales et pouvoirs d'Etat

Peter van der Veer L'Etat moral:

religion, nation et empire dans la Grande-Bretagne victorienne et l'Inde britannique

47. Voir Nicholas Dirks,

"The Conversion of Caste: Location,

Translation, and Appropriation ", in Peter van der Veer (ed.)

Conversion to Modernities.

The Globalization of Christianity,

New York, Routledge, 1995, pp. 115-137.

48. 1824-1883.

49. Kenneth Jones, Arya Dharm, Berkeley, University of California Press, 1976. conséquence de la victoire des envahisseurs. Son argument avait pour fonction de défendre son propre travail de missionnaire dans la population drâvidienne «originelle» en discréditant les prêtres brahmanes. Mais sa théorie d'une race drâvidienne a fini par être utilisée au Sud pour des buts politiques n'ayant plus rien à voir avec la conversion chrétienne $^{47}$. La théorie de la race aryenne a été adoptée en Inde du Nord par le mouvement réformiste le plus important de l'Hindouisme, l'Arya Samaj. Son fondateur, Swami Dayananda Sarasvati ${ }^{48}$, était l'un des nombreux gourous de l'Inde au XIX ${ }^{\mathrm{e}}$ siècle. Il avait été initié dans l'ordre des Dashanamis Shivaites, un ordre ascétique hindou prestigieux, qui n'autorisait que les Brahmanes à faire vœu d'ascétisme. Tout comme d'autres ascètes de son ordre, Dayananda avait voyagé dans toute l'Inde, visitant les lieux sacrés. Il avait plutôt bien réussi et semblait devoir bientôt fonder sa propre communauté de disciples ascétiques et non initiés. En 1872, Dayananda rend visite au chef Brahmo Debendranath Tagore à Calcutta pendant quatre mois. Ce séjour semble avoir transformé son style. Il abandonne ses vêtements ascétiques et renonce à l'usage de l'éloquence sanskrite pour l'hindi ${ }^{49}$. Dayananda ressent déjà profondément, en réformiste qu'il est devenu, la dégénérescence de la religion hindoue et le besoin de la revitaliser. Je me contenterai ici de résumer les points qui font de la religion aryenne (Arya Dharm) de Dayananda un programme religieux radicalement nouveau.

Premièrement, il propose de revenir aux textes védiques et de remplacer les commentaires traditionnels qui en ont été donnés. Il fournit ses propres commentaires de ces textes, en sanskrit, dans lesquels il cherche à montrer que toute la connaissance scientifique de l'Occident était en fait déjà présente dans la révélation védique. Dans la conception évolutionniste du monde au $\mathrm{XIX}^{\mathrm{e}}$ siècle en Europe, le monothéisme est considéré comme la forme la plus élevée de la religion. Une religion doit être monothéiste pour être rationnelle et pour rendre possible une compréhension scientifique du monde. Un deuxième aspect important du programme de Dayananda réside dans ses attaques contre le système des castes, dans lequel il voit une dégénérescence de la hiérarchie naturelle de la société, fondée selon quatre groupes définis par leurs fonctions: les prêtres, les guerriers, les commerçants et les serviteurs. En dépit de l'importance qu'il accorde aux textes sacrés et au rituel brahmanique, Dayananda s'en prend ici 
directement à l'hégémonie des prêtres brahmanes sur les rituels. Il prolonge une réflexion sur les prêtres qui avait ses racines, comme nous l'avons vu avec Rammohan Roy, à la fois dans les débats brahmaniques et dans les attaques coloniales contre les Brahmanes. Mais il convient de souligner la nouveauté radicale du programme de Dayananda: grâce à lui, l'Arya Samaj devient une communauté religieuse dans laquelle tout le pouvoir religieux gravite autour des laïcs. À sa mort, il n'est pas remplacé par un autre gourou, mais par un comité de membres laïques. Un troisième point de ce programme d'innovation réside dans la primauté accordée à l'éducation par l'Arya Samaj. Un grand nombre d'écoles furent fondées dans le Pendjab et ailleurs, attirant encore aujourd'hui beaucoup d'élèves hindous non aryens. Une telle activité sociale a fait de l'Arya Samaj un sérieux concurrent pour les missions chrétiennes. L'Arya Samaj est devenu un important élément dans l'apparition d'un «public» hindou. Ce mouvement présente le débat sur la nature de l'Hindouisme aux masses populaires d'une manière beaucoup plus directe que Rammohan ou Bankim. Le message de Dayananda se développe dans le contexte des importantes traditions discursives hindoues et il est resté proche d'elles. Dayananda était un écrivain et un orateur prolifique, constamment en débat avec d'autres chefs hindous, suivant aussi en cela une vieille tradition de contestation publique de l'opinion religieuse (shastrartha). À la fin de sa vie, il élabore la doctrine révolutionnaire de la protection de la Vache-mère contre les bouchers britanniques et musulmans, qui allait permettre d'assurer la participation des masses à la sphère publique.

\section{Conclusion}

Si beaucoup des thèses défendues dans cet article sont provisoires et préliminaires, cela est encore plus vrai pour la conclusion. J'espère avoir fait comprendre (1) que la religion a joué un rôle crucial dans la constitution de l'Etat-nation moderne en Grande-Bretagne comme en Inde; (2) que les processus de la construction de l'État dans ces deux pays ont été mis en relation par l'intermédiaire de l'Empire; et (3) que la question impériale a modifié le rôle joué par la religion en Grande-Bretagne et en Inde. L'État moderne dépend, selon la théorie libérale, de la formation d'une société civile, composée de 


\section{O S S I E R}

Représentations nationales et pouvoirs d'Etat

Peter van der Veer L'Etat moral:

religion, nation et empire

dans la Grande-Bretagne victorienne et l'Inde britannique
50. Bernard Cohn \& Nicholas Dirks, «Beyond the Fringe: the Nation State, Colonialism and the Technologies of Power ", Journal of Historical Sociology 1, 2, 1988, pp. 224-230. sujets libres mais civilisés, et d'une sphère publique permettant le bon déroulement du débat rationnel. Dans cette théorie, les notions de «liberté» et de «rationalité» sont définies en termes de "laïcité». J'ai essayé de montrer que, contrairement à ce qu'affirme cette théorie, la religion est un lieu majeur de formation des sujets rationnels, moraux, et une dimension essentielle de l'organisation des sphères publiques créées par ces sujets. Ce que les sociétés anti-esclavagistes, les sociétés bibliques, l'agitation anti-catholique, les pétitions anti-sati, les missions Ramakrishna, les mouvements pour la protection de la Vache ont tous en commun, c'est d'avoir contribué à la création de sphères publiques d'interactions qui sont cruciales dans la formation des identités nationales. La teneur morale de ces mouvements est essentielle à la compréhension de la mission de l'Empire, aussi bien qu'à celle de la mission du nationalisme anti-colonial. J'espère avoir aussi démontré que l'hypothèse selon laquelle l'organisation politique britannique serait laique, alors que l'organisation indienne serait religieuse est fausse. J'ai suggéré qu'une distinction nette et structurelle entre nation et État ne pouvait être faite. Dans la période contemporaine, l'État-nation a été construit comme une entité à trait d'union, ce qui signifie que les deux termes sont étroitement liés. Il existe, bien sûr, une conception libérale selon laquelle l'État est à l'extérieur de la société civile et peut être critiqué par celle-ci, dans la mesure où c'est elle qui limite le pouvoir de l'État. Mais il me semble que l'État moderne n'est pas une entité, mais un noyau de projets et de dispositions grâce auquel la société est organisée. Le caractère extérieur de l'État est un effet de ces projets. C'est surtout à travers le projet éducatif et à travers des dispositions légales que le sujet moderne s'est formé. La langue, la race et la religion sont aussi des entités qui ont été construites au cours du processus de formation de l'État-nation. Cela est vrai pour la métropole comme pour la colonie. La mission morale de l'État moderne est d'organiser la santé, la richesse et le bienêtre de ses citoyens et, pour être capable de faire cela, il doit apprendre à les connaître selon divers projets de documentation, tels que le recensement ${ }^{50}$. On peut, peutêtre, mesurer le degré de "religiosité » et de «laïcité » d'une société donnée en examinant dans quelle mesure cette connaissance d'État est rassemblée dans des catégories religieuses et dans quelle mesure la distribution du pouvoir s'effectue par l'intermédiaire des organisations 
religieuses. Il est possible, mais je n'ai pas abordé ce point ici, qu'au $\mathrm{XX}^{\mathrm{e}}$ siècle les Églises et autres organisations religieuses, aient peu à peu perdu l'importance qu'elles ont eue dans l'organisation de l'État-nation en GrandeBretagne, au profit des organisations ouvrières ou des partis politiques. Un tel changement pourrait avoir été favorisé par le rôle de plus en plus grand qu'ont joué les théories scientifiques de la race dans la définition de la nation britannique, comparé au Christianisme. C'est certainement aussi le développement du pouvoir impérial de la Grande-Bretagne dans la seconde moitié du XIX siècle qui a rendu possible les fantasmes de supériorité raciale. En outre, l'apparente neutralité religieuse qui était considérée comme un élément essentiel à la domination impériale (même davantage, peut-être, après la révolte de 1857 qu'avant), a fait de la race un meilleur indicateur de la différence que la religion. Cela a permis au moins à certains membres du gouvernement d'échapper à la pression constante des Évangéliques pour promouvoir le Christianisme en Inde. Cependant, ces velléités impériales de neutralité religieuse (une neutralité qui n'existait pas dans la métropole), n'ont pas empêché les Indiens d'y voir un État fondé sur une moralité clairement chrétienne. Évidemment, la différence cruciale entre l'État moderne dans la métropole et dans la colonie tient au fait que dans le premier cas le projet de légitimité politique s'exprime en termes de "nation», de citoyenneté et d'identité nationale, tandis que dans le dernier cas, les «sujets» sont exclus de la citoyenneté, leur identité nationale étant soit refusée soit dénigrée. Les différences religieuse et raciale sont des moyens de légitimer des différences de pouvoir. C'est pourquoi les nationalismes anti-coloniaux ne sont pas seulement des luttes pour le pouvoir sur la scène politique, mais aussi une façon de s'opposer à l'hégémonie culturelle que véhicule la théorie coloniale de la différence. Ils le font souvent, comme dans les cas examinés ici, en défendant une interprétation alternative des fondements de l'hégémonie, qu'il s'agisse de religion ou de race. Vivekananda affirme la supériorité de la spiritualité de l'Hindouisme sur le matérialisme occidental et contre lui. Ce faisant, il nie que le Christianisme de la Grande-Bretagne possède une moralité supérieure qui justifierait que les Britanniques puissent gouverner l'Inde. L'influence de la Grande-Bretagne ne repose, à ses yeux, que sur des facteurs matériels. Mais ce sont précisément ces facteurs qui ont mis en 


\section{O S S I E R}

Représentations nationales et pouvoirs d'Etat

Peter van der Veer L'Etat moral:

religion, nation et empire

dans la Grande-Bretagne

victorienne et l'Inde britannique péril la dimension spirituelle que le Christianisme britannique aurait pu posséder. Dayananda emprunte la théorie de la race aryenne à l'Orientalisme, mais au lieu d'accepter l'idée selon laquelle le Christianisme avait pour mission d'assurer la rédemption d'une civilisation hindoue dépravée, il propose un retour à la religion védique qui a précédé le Christianisme et qui est à l'origine même de toute moralité.

J'ai examiné dans cet article comment les interactions qui ont produit les notions de spiritualité hindoue et l'Hindouisme aryen ont provoqué non seulement le développement d'un puissant nationalisme hindou tourné contre l'oppression coloniale, mais aussi l'exclusion des Musulmans de la nation. Dans une période au cours de laquelle les Catholiques en Grande-Bretagne ont été progressivement incités à devenir partie intégrante de la nation, en Inde, les Hindous et les Musulmans ont créé leurs propres sphères publiques séparées, dans un contexte de neutralité religieuse et d'extériorité de l'État colonial. Cette évolution s'explique, d'après moi, par la façon dont se sont structurées les relations sociales dans le monde colonial. Mais c'est une hypothèse que j'examinerai de façon plus approfondie dans une phase ultérieure de cette recherche.

Traduction de Sylvie Bach 